\title{
Clinical Presentation in Hypertension; an Observational Comparative Study to Determine Features in Male and Female Patients
}

\author{
Ali ${ }^{1}$, Zareen $I^{2}$, Zaidi SIH ${ }^{3}$, Samreen $F^{4}$,Madiha $A^{5}$, Syed Muhammad ZHN ${ }^{6}$, Neeta $\mathbf{M}^{7 *}$, Muhammad ${ }^{8}$, \\ Hamza J ${ }^{9}$ and Adnan $\mathbf{A}^{10}$ \\ ${ }^{1} M B B S, D H Q$ Teaching Hospital, Gujranwala, Pakistan
}

${ }^{2}$ MBBS, FCPS, Assistant Professor, Department of Pathology, Jinnah Sindh Medical University, Karachi, Pakistan

${ }^{3}$ MBBS, M.Phil, Associate Professor, Department of Pharmacology, Bahria University, Karachi, Pakistan

${ }^{4}$ MBBS, Medical officer, Jinnah Sindh Medical University, Karachi, Pakistan

${ }^{5}$ MBBS, Dow University of Health Sciences, Karachi, Pakistan

${ }^{6}$ MBBS, MSBE Assistant Professor, Department of Community Medicine, Baqai Medical University, Karachi, Pakistan

${ }^{7}$ Manager Medical Affairs and Clinical Research, Hilton PharmaPvt ltd, Pakistan

${ }^{8}$ Resident, Memon Hospital, Karachi, Pakistan

${ }^{9}$ MBBS, Medical Officer, Hamdard University Hospital, Karachi, Pakistan

${ }^{10}$ MBBS, Senior Lecturer, Department of Physiology, Altibri Medical College Karachi, Karachi, Pakistan

Received: September 19, 2017; Accepted: September 26, 2017; Published: October 24, 2017

*Corresponding author: Neeta Maheshwary, Manager Medical Affairs and Clinical Research, Hilton Pharma Pvt ltd, Pakistan,

E-mail: neeta_maheshwary@yahoo.com

\begin{abstract}
Background: Hypertension is regarded as an important public health challenge worldwide. It is the most important modifiable risk factor for cardiovascular, cerebrovascular and renal disease and has been identified as the leading global risk factor for mortality and as the third leading risk factor for disease burden.

Objective: To identify and compare the signs and symptoms of hypertension among males and females in a Pakistani population.

Methods: A multicenter cross-sectional study was carried out among patients with self-reported history of hypertension. A total of 160 patients aged 18 or above were included in the study by using convenient sampling. Each patient's demographic profile and the history of hypertension associated medical conditions were recorded through interview. Blood pressure was measured with the help of Sphygmomanometer using Stethoscope. The total study duration was 6 months.

Results: A higher percentage of females had both systolic and diastolic hypertension than males $(85.0 \%$ vs. $80.0 \%$ and $82.5 \%$ vs. $76.3 \%$ respectively).The site of headache had positive correlation with both systolic and diastolic blood pressures in males ( $p=0.04$ and $p=0.007$ respectively) as well as females ( $\mathrm{p}<0.001$ for both). Both the severity of headache and severity of dyspnea were positively correlated with diastolic blood pressure in males ( $\mathrm{p}=0.006$ and $\mathrm{p}=0.018$ respectively) whereas the type of vision problem was negatively correlated with diastolic blood pressure in females $(\mathrm{p}=0.021)$.Among males the clinical history of headache $(\mathrm{p}=0.018)$, vertigo $(\mathrm{p}=0.016)$ and dyspnea $(\mathrm{p}=0.004)$ were significantly associated with systolic blood pressure whereas among females only age $(\mathrm{p}=0.048)$ was significantly associated with diastolic blood pressure.
\end{abstract}

Conclusion: The symptoms significantly associated with systolic or diastolic hypertension included age, clinical history of headache, its site and severity, vertigo, dyspnea and its severity and type of vision problem although a disparity was found among both genders.

Keywords: Clinical Presentation; Hypertension; Male and Female Patients 


\section{Introduction}

Because of its high prevalence and concomitant increase in risk of disease, hypertension is worldwide regarded as an important public health challenge. [1,2] It is the most important modifiable risk factor for cardiovascular, cerebrovascular and renal disease. The comparative Risk Assessment Collaborating Group has identified hypertension as the leading global risk factor for mortality and as the third leading risk factor for disease burden. [3] An analysis indicates that more than a quarter of the world's adult population-totaling nearly one billionhad hypertension in the year 2000, and that this proportion will increase to $29 \%-1.56$ billion-by the year 2025 . It also suggests that men and women have similar overall prevalence of hypertension, and that such prevalence increase with age consistently in all world regions. [4] The projections for the year 2025 are based on the assumption that the country, age, and sex specific prevalence estimates will remain constant. [5] According to the World Health Organization, the total prevalence of high blood pressure in Pakistan is estimated to be $25.2 \%$ (25.6\% in males and $24.8 \%$ in females). [6]

Hypertension is defined as a systolic blood pressure (SBP) of $140 \mathrm{~mm} \mathrm{Hg}$ or more, or a diastolic blood pressure (DBP) of 90 mmHg or more or taking antihypertensive medication. [7] There are two types of hypertension namely essential and secondary. Essential hypertension can be defined as a rise in blood pressure of unknown cause that increases risk for cerebral, cardiac, and renal events. [8] Secondary hypertension is defined as increased systemic blood pressure due to an identifiable cause. Only 5-10\% of patients suffering from arterial hypertension have a secondary form, whereas the vast majority has essential (idiopathic or primary) hypertension. [9] The clinical presentation of high blood pressure differs from person to person. Usually it causes no signs and symptoms and that is why it is referred as a "silent killer."

Based on the recommendations of the Seventh Report of the Joint National Committee on Prevention, Detection, Evaluation, and Treatment of High Blood Pressure (JNC 7), BP for adults aged 18 years or older has been classified into four categories as normal, prehypertension, stage 1 and stage 2. Normalsystolic $<120 \mathrm{mmHg}$; diastolic $<80 \mathrm{~mm} \mathrm{Hg}$. Prehypertensionsystolic 120-139 mmHg; diastolic 80-89 mm Hg. Stage 1- systolic 140-159 mmHg; diastolic 90-99 mm Hg. Stage 2-160 mmHg or greater; diastolic $100 \mathrm{mmHg}$ or greater. [10]

The WHO recognizes three levels of hypertension as levels 1 , 2 and 3.

Level 1(mild hypertension) means a systolic BP between 140 and $159 \mathrm{~mm} \mathrm{Hg}$ and a diastolic BP between 90 and 99, level 2 (medium-severe hypertension) means a systolic BP between 160 and $179 \mathrm{~mm} \mathrm{Hg}$ and a diastolic BP between 100-109 mm Hg whereas level 3 means a systolic BP of 180 and above and a diastolic BP of $110 \mathrm{mmHg}$ and above.

Literature search does not reveal ample data are on comparison of signs and symptoms of hypertension among male and female patients in Pakistan. This study therefore was intended to gender wise identify and compare the signs and symptoms of hypertension in a Pakistani population thereby aiding in prioritization of hypertension risk management in each gender.

\section{Material and Methods}

A multicenter cross-sectional study was carried out among patients with self-reported history of hypertension. After taking ethical approval from ethical review committee of Urban Hospital, Karachi. A total of 160 patients aged 18 or above were included in the study by using convenient sampling. Patients with history of diabetes, cardiac events, neurological disorders, cluster headache, gastrointestinal disease, visual problems, epistaxis before diagnosed with hypertension and morbid obesity were excluded from the study.

Each patient's demographic profile and the history of hypertension associated medical conditions were recorded through interview such as duration of hypertension, site, duration and severity of headache, presence of chest pain and vertigo and their severity, presence of edema, its laterality and grading and presence of vision problems and their classification according to loss in the field of vision. Few additional symptoms such as nausea, sleep apnea, palpitations, fatigue and confusion were also assessed subjectively. Blood pressure was measured with the help of Sphygmomanometer using Stethoscope. The duration of the study was 6 months.

\section{Results}

The study results showed that the mean age of male patients was slightly higher than that of females $(49.99 \pm 11.73$ years vs. $42.93 \pm 13.50$ years) and therefore a higher percentage of them was above 40 years of age ( $77.5 \%$ vs. $47.5 \%)$. A higher percentage of females had both systolic and diastolic hypertension than males ( $85.0 \%$ vs. $80.0 \%$ and $82.5 \%$ vs. $76.3 \%$ respectively). Both the mean systolic and diastolic blood pressures were higher in females (148.29 $\pm 15.80 \mathrm{mmHg}$ vs. $145.11 \pm 15.98 \mathrm{mmHg}$ and $92.14 \pm 7.55$ mmHg vs. $91.62 \pm 9.81 \mathrm{mmHg}$ respectively). The males had higher mean duration of hypertension than females $(4.68 \pm 3.55$ years vs. $4.34 \pm 3.17$ years) and a much higher percentage of them were smokers as well (20.0\% vs. 3.8\%). The clinical history of headache was more prevalent among females $(60.0 \%$ vs. $45.0 \%)$ whereas its most common site was occipital in males and temporal and females ( $41.7 \%$ and $33.3 \%$ respectively). A majority of both male and female patients reported their headache to be of moderate severity (55.6\% and $43.8 \%$ respectively). A higher percentage of females gave a positive history of vertigo $(40.0 \%$ vs. $31.3 \%)$ whereas a majority of both males and females reported their vertigo to be mild in severity $(73.1 \%$ and $65.6 \%$ respectively). The prevalence of edema and its laterality were similar in both genders though unlike males a majority $(57.1 \%)$ of females reported it to be moderate in severity. The prevalence of chest pain was also similar $(25.0 \%)$ in both genders though a higher percentage of females required hospital visit for its treatment (21.1\% vs. $10.0 \%)$. A higher percentage of females gave a positive history of vision problems $(50.0 \%$ vs. $38.8 \%)$ whereas the most common type of vision problem in both males and females was 


\begin{tabular}{|c|c|c|c|}
\hline \multirow{2}{*}{\multicolumn{2}{|c|}{ Variables }} & Males $(n=80)$ & Females $(n=80)$ \\
\hline & & Frequency $(\%) /$ Mean \pm S.D. & Frequency $(\%) /$ Mean \pm S.D. \\
\hline \multicolumn{2}{|c|}{ Age (Years) } & $49.99 \pm 11.73$ & $42.93 \pm 13.50$ \\
\hline \multirow{2}{*}{ Age Group } & $\leq 40$ Years & $18(22.5)$ & $42(52.5)$ \\
\hline & $>40$ Years & $62(77.5)$ & $38(47.5)$ \\
\hline \multirow{2}{*}{ Systolic Blood Pressure } & Normotensive/ Prehypertensive & $16(20.0)$ & $12(15.0)$ \\
\hline & Stage 1/Stage 2 Hypertension & $64(80.0)$ & $68(85.0)$ \\
\hline \multirow{2}{*}{ Diastolic Blood Pressure } & Normotensive/ Prehypertensive & $19(23.8)$ & $14(17.5)$ \\
\hline & Stage 1 /Stage 2 Hypertension & $61(76.3)$ & $66(82.5)$ \\
\hline \multicolumn{2}{|c|}{ Systolic Blood Pressure (mm Hg) } & $145.11 \pm 15.98$ & $148.29 \pm 15.80$ \\
\hline \multicolumn{2}{|c|}{ Diastolic Blood Pressure (mm Hg) } & $91.62 \pm 9.81$ & $92.14 \pm 7.55$ \\
\hline \multicolumn{2}{|c|}{ Hypertension Duration (Years) } & $4.68 \pm 3.55$ & $4.34 \pm 3.17$ \\
\hline \multirow{2}{*}{ Smoking } & Yes & $16(20.0)$ & $3(3.8)$ \\
\hline & No & $64(80.0)$ & $77(96.3)$ \\
\hline \multirow{2}{*}{$\begin{array}{c}\text { Clinical History of } \\
\text { Headache }\end{array}$} & Yes & $36(45.0)$ & $48(60.0)$ \\
\hline & No & $44(55.0)$ & $32(40.0)$ \\
\hline \multirow{5}{*}{ Site of Heacache1 } & Temporal & $4(11.1)$ & $16(33.3)$ \\
\hline & Parietal & $5(13.9)$ & $6(12.5)$ \\
\hline & Occipital & $15(41.7)$ & $10(20.8)$ \\
\hline & Frontal & $4(11.1)$ & $1(2.1)$ \\
\hline & Complete & $8(22.2)$ & $15(31.3)$ \\
\hline \multirow{3}{*}{ Severity of Headache1 } & Mild & $5(13.9)$ & $9(18.8)$ \\
\hline & Moderate & $20(55.6)$ & $21(43.8)$ \\
\hline & Severe & $11(30.6)$ & $18(37.5)$ \\
\hline \multirow{2}{*}{ Vertigo } & Yes & $25(31.3)$ & $32(40.0)$ \\
\hline & No & $55(68.7)$ & $48(60.0)$ \\
\hline \multirow{3}{*}{ Severity of Vertigo 2} & Mild & $19(73.1)$ & $21(65.6)$ \\
\hline & Moderate & $5(19.2)$ & $10(31.3)$ \\
\hline & Severe & $2(7.7)$ & $1(3.1)$ \\
\hline \multirow{2}{*}{ Edema } & Yes & $19(23.8)$ & $21(26.3)$ \\
\hline & No & $61(76.3)$ & $59(73.7)$ \\
\hline \multirow{2}{*}{ Laterality of Edema3 } & Unilateral & $12(63.2)$ & $13(61.9)$ \\
\hline & Bilateral & $7(36.8)$ & $8(38.1)$ \\
\hline $\begin{array}{c}\text { Grading of Bilateral } \\
\text { Edema3 }\end{array}$ & Mild & $5(26.3)$ & $4(19.0)$ \\
\hline \multirow{2}{*}{$\begin{array}{c}\text { Grading of Bilateral } \\
\text { Edema3 }\end{array}$} & Mild & $5(26.3)$ & $4(19.0)$ \\
\hline & Moderate & $7(36.8)$ & $12(57.1)$ \\
\hline
\end{tabular}




\begin{tabular}{|c|c|c|c|}
\hline \multirow{3}{*}{ Chest Pain } & Severe & $7(36.8)$ & $5(23.8)$ \\
\hline & Yes & $20(25.0)$ & $20(25.0)$ \\
\hline & No & $60(75.0)$ & $60(75.0)$ \\
\hline \multirow{3}{*}{ Severity of Chest Pain4 } & Improves with rest & $8(40.0)$ & $5(26.3)$ \\
\hline & Needs pain relieving medication & $10(50.0)$ & $10(52.6)$ \\
\hline & Requires hospital visit & $2(10.0)$ & $4(21.1)$ \\
\hline \multirow{2}{*}{ Vision Problems } & Yes & $31(38.8)$ & $40(50.0)$ \\
\hline & No & $49(61.2)$ & $40(50.0)$ \\
\hline \multirow{4}{*}{ Type of Vision Problem5 } & Loss of Central Vision & $5(17.2)$ & $3(7.9)$ \\
\hline & Loss of Peripheral Vision & $5(17.2)$ & $13(34.2)$ \\
\hline & Dark Spots in field of Vision & $11(37.9)$ & $16(42.1)$ \\
\hline & Pain in Eyes & $8(27.6)$ & $6(15.8)$ \\
\hline \multirow{2}{*}{ Dyspnea } & Yes & $30(37.5)$ & $37(46.3)$ \\
\hline & No & $50(62.5)$ & $43(53.8)$ \\
\hline \multirow{3}{*}{ Dyspnea Severity6 } & Mild & $6(20.0)$ & $6(16.2)$ \\
\hline & Moderate & $14(46.7)$ & 18(48.6) \\
\hline & Severe & $10(33.3)$ & $13(35.1)$ \\
\hline \multirow{2}{*}{ Nausea7 } & Yes & $14(17.7)$ & $16(20.0)$ \\
\hline & No & $65(82.3)$ & $64(80.0)$ \\
\hline \multirow{2}{*}{ Sleep Apnea } & Yes & $37(46.3)$ & $33(41.3)$ \\
\hline & No & $43(53.7)$ & $47(58.7)$ \\
\hline \multirow{2}{*}{$\begin{array}{c}\text { Irregular Heartbeat/ } \\
\text { Palpitation }\end{array}$} & Yes & $28(35.0)$ & $30(37.5)$ \\
\hline & No & $52(65.0)$ & $50(62.5)$ \\
\hline \multirow{2}{*}{ Fatigue7 } & Yes & $52(65.8)$ & $48(60.0)$ \\
\hline & No & $27(34.2)$ & $32(40.0)$ \\
\hline \multirow{2}{*}{ Confusion 7} & Yes & $39(49.4)$ & $41(51.3)$ \\
\hline & No & $40(50.6)$ & $39(48.7)$ \\
\hline \multicolumn{4}{|c|}{${ }^{1} \mathrm{n}=36$ and 48 in males and females respectively } \\
\hline \multicolumn{4}{|c|}{${ }^{2} n=26$ and 32 in males and females respectively } \\
\hline \multicolumn{4}{|c|}{${ }^{3} \mathrm{n}=19$ and 21 in males and females respectively } \\
\hline \multicolumn{4}{|c|}{${ }^{4} n=20$ and 19 in males and females respectively } \\
\hline \multicolumn{4}{|c|}{${ }^{5} n=29$ and 38 in males and females respectively } \\
\hline \multicolumn{4}{|c|}{${ }^{6} \mathrm{n}=30$ and 37 in males and females respectively } \\
\hline${ }^{7} n=79$ and 80 in males and fen & respectively & & \\
\hline
\end{tabular}

dark spots in field of vision (37.9\% and $42.1 \%$ respectively). A higher percentage of females gave a positive history of dyspnea (46.3\% vs. $37.5 \%)$ whereas a similar majority of both genders reported it to be moderate in severity. A similar percentage of both genders were also suffering from nausea, sleep apnea, irregular heartbeat/palpitation, fatigue and vertigo [Table 1].

The study analysis also revealed that the site of headache had positive correlation with both systolic and diastolic blood pressures in males ( $\mathrm{p}=0.04$ and $\mathrm{p}=0.007$ respectively) as well as females $(\mathrm{p}<0.001$ for both). Both the severity of headache and severity of dyspnea were found to be positively correlated with diastolic blood pressure in males only $(\mathrm{p}=0.006$ and $\mathrm{p}=0.018$ respectively) whereas the type of vision problem was negatively correlated with diastolic blood pressure in females only $(p=0.021)$. None of the other variables were significantly correlated with either systolic or diastolic blood pressure in both genders [Table 2]. 
Table 2: Correlation between blood pressure and patients' signs and symptoms: Gender wise comparison

\begin{tabular}{|c|c|c|c|c|c|c|c|c|}
\hline \multirow{3}{*}{ Variables } & \multicolumn{4}{|c|}{ Males $(n=80)$} & \multicolumn{4}{|c|}{ Females $(n=80)$} \\
\hline & \multicolumn{2}{|c|}{ SBP } & \multicolumn{2}{|c|}{ DBP } & \multicolumn{2}{|c|}{ SBP } & \multicolumn{2}{|c|}{ DBP } \\
\hline & $\rho$ & $\mathbf{p}$ & $\rho$ & $\mathbf{P}$ & $\rho$ & $\mathbf{P}$ & $\rho$ & $\mathbf{P}$ \\
\hline Age (Years) & -0.045 & 0.692 & -0.171 & 0.128 & -0.01 & 0.929 & -0.166 & 0.141 \\
\hline Age Groups & 0.059 & 0.606 & 0.022 & 0.844 & -0.003 & 0.977 & -0.147 & 0.193 \\
\hline $\begin{array}{c}\text { Duration of } \\
\text { Hypertension (Years) }\end{array}$ & 0.017 & 0.882 & -0.017 & 0.88 & -0.1 & 0.379 & -0.031 & 0.788 \\
\hline Site of Headache & $0.344^{*}$ & 0.04 & $0.44^{*}$ & 0.007 & $0.53^{*}$ & $<0.001$ & $0.563^{*}$ & $<0.001$ \\
\hline Severity of Headache & 0.244 & 0.152 & $0.448^{*}$ & 0.006 & 0.019 & 0.9 & 0.085 & 0.568 \\
\hline Severity of Vertigo & -0.095 & 0.644 & 0.172 & 0.401 & 0.162 & 0.376 & 0.327 & 0.068 \\
\hline Laterality of Edema & 0.03 & 0.903 & 0.224 & 0.356 & -0.016 & 0.944 & 0.074 & 0.75 \\
\hline $\begin{array}{c}\text { Grading of Bilateral } \\
\text { Edema }\end{array}$ & -0.087 & 0.723 & -0.065 & 0.79 & -0.113 & 0.625 & -0.086 & 0.712 \\
\hline $\begin{array}{c}\text { Severity of Chest } \\
\text { Pain }\end{array}$ & -0.101 & 0.671 & -0.084 & 0.724 & 0.061 & 0.803 & -0.044 & 0.858 \\
\hline $\begin{array}{c}\text { Type of Vision } \\
\text { Problem }\end{array}$ & -0.087 & 0.653 & 0.101 & 0.603 & -0.098 & 0.559 & $-0.373^{*}$ & 0.021 \\
\hline Severity of Dyspnea & 0.206 & 0.274 & $0.429 *$ & 0.018 & 0.125 & 0.459 & 0.02 & 0.907 \\
\hline
\end{tabular}

The study findings further showed that among males the clinical history of headache $(\mathrm{p}=0.018)$, vertigo $(\mathrm{p}=0.016)$ and dyspnea $(\mathrm{p}=0.004)$ were significantly associated with systolic blood pressure where those who had stage 1 /stage 2 systolic hypertension were more likely to have positive clinical history of headache, to have vertigo and to have dyspnea than those who did not $(51.6 \%$ vs. $18.8 \%, 37.5 \%$ vs. $6.2 \%$ and $45.3 \%$ vs. $6.2 \%$ respectively)whereas among females only age ( $\mathrm{p}=0.048)$ was significantly associated with diastolic blood pressure where those who had stage 1/stage 2 diastolic hypertension were more likely to be $\leq 40$ years of age than those who did not $(57.6 \%$ vs. $28.6 \%$ respectively). None of the other variables were found to be significantly associated with either diastolic blood pressure among males or systolic blood pressure among females [Tables $3,4]$.

Table 3: Relationship between blood pressure and patients' signs and symptoms in males

\begin{tabular}{|c|c|c|c|c|c|}
\hline \multirow{4}{*}{ Variables } & & \multicolumn{4}{|c|}{ Males $(n=80)$} \\
\hline & & \multicolumn{2}{|c|}{ SBP } & \multicolumn{2}{|c|}{ DBP } \\
\hline & & $\begin{array}{c}\text { Normotensive/ } \\
\text { Prehypertensive }\end{array}$ & $\begin{array}{c}\text { Stage 1/ Stage } 2 \\
\text { Hypertension }\end{array}$ & $\begin{array}{c}\text { Normotensive/ } \\
\text { Prehypertensive }\end{array}$ & $\begin{array}{c}\text { Stage 1/Stage } 2 \\
\text { Hypertension }\end{array}$ \\
\hline & & Frequency (\%) & Frequency (\%) & Frequency (\%) & Frequency (\%) \\
\hline Age Group & $>40$ Years & $15(93.8)$ & $47(73.4)$ & $17(89.5)$ & $45(73.8)$ \\
\hline \multicolumn{2}{|c|}{$\mathbf{P}$} & \multicolumn{2}{|c|}{$0.103^{*}$} & \multicolumn{2}{|c|}{$0.214^{*}$} \\
\hline \multirow{2}{*}{ Smoking } & Yes & $3(18.8)$ & $13(20.3)$ & $4(21.1)$ & $12(19.7)$ \\
\hline & No & $13(81.2)$ & $51(79.7)$ & $15(78.9)$ & $49(80.3)$ \\
\hline \multicolumn{2}{|c|}{$\mathbf{P}$} & \multicolumn{2}{|c|}{$>0.999 *$} & \multicolumn{2}{|c|}{$>0.999 *$} \\
\hline \multirow{2}{*}{$\begin{array}{c}\text { Clinical History of } \\
\text { Headache }\end{array}$} & Yes & $3(18.8)$ & $33(51.6)$ & $6(31.6)$ & $30(49.2)$ \\
\hline & No & $13(81.2)$ & $31(48.4)$ & $13(68.4)$ & $31(50.8)$ \\
\hline \multicolumn{2}{|c|}{$\mathbf{P}$} & \multicolumn{2}{|c|}{0.018} & \multicolumn{2}{|c|}{0.178} \\
\hline
\end{tabular}




\begin{tabular}{|c|c|c|c|c|c|}
\hline \multirow{2}{*}{ Severity of Headache } & Mild/Moderate & $3(100)$ & $22(66.7)$ & $6(100)$ & $19(63.3)$ \\
\hline & Severe & Nil & $11(33.3)$ & Nil & $11(36.7)$ \\
\hline \multicolumn{2}{|c|}{$\mathbf{P}$} & \multicolumn{2}{|c|}{$0.538^{*}$} & \multicolumn{2}{|c|}{$0.148^{*}$} \\
\hline \multirow{2}{*}{ Vertigo } & Yes & $1(6.2)$ & $24(37.5)$ & $3(15.8)$ & $22(36.1)$ \\
\hline & No & 15(93.8) & $40(62.5)$ & $16(84.2)$ & $39(63.9)$ \\
\hline \multicolumn{2}{|c|}{$\mathbf{P}$} & \multicolumn{2}{|c|}{0.016} & \multicolumn{2}{|c|}{0.096} \\
\hline \multirow{2}{*}{ Edema } & Yes & $1(6.2)$ & $18(28.1)$ & $2(10.5)$ & $17(27.9)$ \\
\hline & No & $15(93.8)$ & $46(71.9)$ & $17(89.5)$ & $44(72.1)$ \\
\hline \multicolumn{2}{|c|}{$\mathbf{P}$} & \multicolumn{2}{|c|}{$0.1^{*}$} & \multicolumn{2}{|c|}{$0.215^{*}$} \\
\hline \multirow{2}{*}{ Laterality of Edema } & Unilateral & $1(100)$ & $11(61.1)$ & $2(100)$ & $10(58.8)$ \\
\hline & Bilateral & Nil & $7(38.9)$ & Nil & $7(41.2)$ \\
\hline \multicolumn{2}{|c|}{$\mathbf{P}$} & \multicolumn{2}{|c|}{$>0.999^{*}$} & \multicolumn{2}{|c|}{$0.509 *$} \\
\hline \multirow{2}{*}{ Chest Pain } & Yes & $1(6.2)$ & 19(29.7) & $3(15.8)$ & $17(27.9)$ \\
\hline & No & $15(93.8)$ & $45(70.3)$ & $16(84.2)$ & $44(72.1)$ \\
\hline \multicolumn{2}{|c|}{$\mathbf{P}$} & \multicolumn{2}{|c|}{$0.060^{*}$} & \multicolumn{2}{|c|}{$0.373^{*}$} \\
\hline \multirow{2}{*}{ Vision Problems } & Yes & $4(25.0)$ & $27(42.2)$ & $5(26.3)$ & $26(42.6)$ \\
\hline & No & $12(75.0)$ & $37(57.8)$ & $14(73.7)$ & $35(57.4)$ \\
\hline \multicolumn{2}{|c|}{$\mathbf{P}$} & \multicolumn{2}{|c|}{0.207} & \multicolumn{2}{|c|}{0.203} \\
\hline \multirow{2}{*}{ Dyspnea } & Yes & $1(6.2)$ & $29(45.3)$ & $5(26.3)$ & $25(41.0)$ \\
\hline & No & $15(93.8)$ & $35(54.7)$ & $14(73.7)$ & $36(59.0)$ \\
\hline \multicolumn{2}{|c|}{$\mathbf{P}$} & \multicolumn{2}{|c|}{0.004} & & \\
\hline Sounituf Drcnmo & Mild/Moderate & $1(100)$ & $19(65.5)$ & $5(100)$ & $15(60.0)$ \\
\hline & Severe & Nil & $10(34.5)$ & Nil & $10(40.0)$ \\
\hline & & & & & \\
\hline & Yes & $1(6.2)$ & $13(20.6)$ & $1(5.3)$ & $13(21.7)$ \\
\hline & No & $15(93.8)$ & $50(79.4)$ & 18(94.7) & $47(78.3)$ \\
\hline & & & & & \\
\hline Sloon Anmon & Yes & $6(37.5)$ & $31(48.4)$ & $9(47.4)$ & $28(45.9)$ \\
\hline 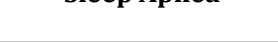 & No & $10(62.5)$ & $33(51.6)$ & $10(52.6)$ & $33(54.1)$ \\
\hline & & & & & \\
\hline Irregular Heartbeat/ & Yes & $3(18.8)$ & $25(39.1)$ & $4(21.1)$ & $24(39.3)$ \\
\hline Paiptitaivil & No & $13(81.2)$ & $39(60.9)$ & $15(78.9)$ & $37(60.7)$ \\
\hline & & & & & \\
\hline Fotimu & Yes & $9(60.0)$ & $43(67.2)$ & $11(61.1)$ & $41(67.2)$ \\
\hline 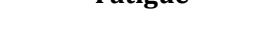 & No & $6(40.0)$ & $21(32.8)$ & $7(38.9)$ & $20(32.8)$ \\
\hline & & & & & \\
\hline Confusion & Yes & $6(40.0)$ & $33(51.6)$ & $10(55.6)$ & $29(47.5)$ \\
\hline 年 & No & $9(60.0)$ & $31(48.4)$ & $8(44.4)$ & $32(52.5)$ \\
\hline & & & & & \\
\hline
\end{tabular}




\begin{tabular}{|c|c|c|c|c|c|}
\hline \multirow{3}{*}{ Variables } & & \multicolumn{4}{|c|}{ Females $(n=80)$} \\
\hline & \multicolumn{3}{|c|}{ SBP } & \multicolumn{2}{|c|}{ DBP } \\
\hline & & $\begin{array}{l}\text { Normotensive/ } \\
\text { Prehypertensive }\end{array}$ & $\begin{array}{c}\text { Stage } 1 / \text { Stage } 2 \\
\text { Hypertension }\end{array}$ & $\begin{array}{l}\text { Normotensive/ } \\
\text { Prehypertensive }\end{array}$ & $\begin{array}{c}\text { Stage } 1 / \text { Stage } 2 \\
\text { Hypertension }\end{array}$ \\
\hline & & Frequency (\%) & Frequency (\%) & Frequency (\%) & Frequency (\%) \\
\hline \multirow{2}{*}{ Age Group } & $\leq 40$ Years & $4(33.3)$ & $38(55.9)$ & $4(28.6)$ & $38(57.6)$ \\
\hline & $>40$ Years & $8(66.7)$ & $30(44.1)$ & $10(71.4)$ & $28(42.4)$ \\
\hline \multicolumn{2}{|c|}{$\mathbf{P}$} & \multicolumn{2}{|c|}{0.149} & \multicolumn{2}{|c|}{0.048} \\
\hline \multirow{2}{*}{ Smoking } & Yes & Nil & $3(4.4)$ & $1(7.1)$ & $2(3.0)$ \\
\hline & No & $12(100)$ & $65(95.6)$ & 13(92.9) & $64(97.0)$ \\
\hline \multicolumn{2}{|c|}{$\mathbf{P}$} & \multicolumn{2}{|c|}{$>0.999^{*}$} & \multicolumn{2}{|c|}{$0.443^{*}$} \\
\hline \multirow{2}{*}{ Clinical History of Headache } & Yes & $9(75.0)$ & $39(57.4)$ & $9(64.3)$ & $39(59.1)$ \\
\hline & No & $3(25.0)$ & $29(42.6)$ & $5(35.7)$ & $27(40.9)$ \\
\hline \multicolumn{2}{|c|}{$\mathbf{P}$} & \multicolumn{2}{|c|}{$0.344^{*}$} & \multicolumn{2}{|c|}{0.719} \\
\hline \multirow{2}{*}{ Severity of Headache } & Mild/Moderate & $5(55.6)$ & $25(64.1)$ & $5(55.6)$ & $25(64.1)$ \\
\hline & Severe & $4(44.4)$ & $14(35.9)$ & $4(44.4)$ & $14(35.9)$ \\
\hline \multicolumn{2}{|c|}{$\mathbf{P}$} & \multicolumn{2}{|c|}{$0.711^{*}$} & \multicolumn{2}{|c|}{$0.711^{*}$} \\
\hline \multirow{2}{*}{ Vertigo } & Yes & $4(33.3)$ & $28(41.2)$ & $7(50.0)$ & $25(37.9)$ \\
\hline & No & $8(66.7)$ & $40(58.8)$ & $7(50.0)$ & $41(62.1)$ \\
\hline \multicolumn{2}{|c|}{$\mathbf{P}$} & \multicolumn{2}{|c|}{$0.754^{*}$} & \multicolumn{2}{|c|}{0.4} \\
\hline \multirow{2}{*}{ Edema } & Yes & $3(25.0)$ & $18(26.5)$ & $4(28.6)$ & $17(25.8)$ \\
\hline & No & $9(75.0)$ & $50(73.5)$ & $10(71.4)$ & $49(74.2)$ \\
\hline \multicolumn{2}{|c|}{$\mathbf{P}$} & \multicolumn{2}{|c|}{$>0.999^{*}$} & \multicolumn{2}{|c|}{$>0.999^{*}$} \\
\hline I atorality of Edoma & Unilateral & $1(33.3)$ & $12(66.7)$ & $2(50.0)$ & $11(64.7)$ \\
\hline & Bilateral & $2(66.7)$ & $6(33.3)$ & $2(50.0)$ & $6(35.3)$ \\
\hline & & & & & \\
\hline$C_{1}$ & Yes & $2(16.7)$ & $18(26.5)$ & $3(21.4)$ & $17(25.8)$ \\
\hline Chest ram & No & $10(83.3)$ & $50(73.5)$ & 11(78.6) & $49(74.2)$ \\
\hline & & & & & \\
\hline W & Yes & $6(50.0)$ & $34(50.0)$ & $9(64.3)$ & $31(47.0)$ \\
\hline & No & $6(50.0)$ & $34(50.0)$ & $5(35.7)$ & $35(53.0)$ \\
\hline & & & & & \\
\hline Dra & Yes & $4(33.3)$ & $33(48.5)$ & $8(57.1)$ & $29(43.9)$ \\
\hline Dyspned & No & $8(66.7)$ & $35(51.5)$ & $6(42.9)$ & $37(56.1)$ \\
\hline & & & & & \\
\hline Severity of Dysnnea & Mild/Moderate & $4(100)$ & $20(60.6)$ & $6(75.0)$ & $18(62.1)$ \\
\hline & Severe & Nil & $13(39.4)$ & $2(25.0)$ & $11(37.9)$ \\
\hline
\end{tabular}



Study to Determine Features in Male and Female Patients

\begin{tabular}{|c|c|c|c|c|c|}
\hline \multicolumn{2}{|c|}{$\mathbf{P}$} & \multicolumn{2}{|c|}{$0.276^{*}$} & \multicolumn{2}{|c|}{$0.685^{*}$} \\
\hline \multirow{2}{*}{ Nausea } & Yes & $2(16.7)$ & $14(20.6)$ & $2(14.3)$ & $14(21.2)$ \\
\hline & No & $10(83.3)$ & $54(79.4)$ & $12(85.7)$ & $52(78.8)$ \\
\hline \multicolumn{2}{|c|}{$\mathbf{P}$} & \multicolumn{2}{|c|}{$>0.999^{*}$} & \multicolumn{2}{|c|}{$0.724^{*}$} \\
\hline \multirow{2}{*}{ Sleep Apnea } & Yes & $3(25.0)$ & $30(44.1)$ & $4(28.6)$ & $29(43.9)$ \\
\hline & No & $9(75.0)$ & $38(55.9)$ & $10(71.4)$ & $37(56.1)$ \\
\hline \multicolumn{2}{|c|}{$\mathbf{P}$} & \multicolumn{2}{|c|}{$0.341^{*}$} & \multicolumn{2}{|c|}{0.289} \\
\hline \multirow{2}{*}{$\begin{array}{c}\text { Irregular Heartbeat/ } \\
\text { Palpitation }\end{array}$} & Yes & $4(33.3)$ & $26(38.2)$ & $6(42.9)$ & $24(36.4)$ \\
\hline & No & $8(66.7)$ & $42(61.8)$ & $8(57.1)$ & $42(63.6)$ \\
\hline \multicolumn{2}{|c|}{$\mathbf{P}$} & \multicolumn{2}{|c|}{$>0.999^{*}$} & \multicolumn{2}{|c|}{0.649} \\
\hline \multirow{2}{*}{ Fatigue } & Yes & $8(66.7)$ & $40(58.8)$ & $11(78.6)$ & $37(56.1)$ \\
\hline & No & $4(33.3)$ & $28(41.2)$ & $3(21.4)$ & $29(43.9)$ \\
\hline \multicolumn{2}{|c|}{$\mathbf{P}$} & \multicolumn{2}{|c|}{$0.754^{*}$} & \multicolumn{2}{|c|}{0.118} \\
\hline \multirow{2}{*}{ Confusion } & Yes & $5(41.7)$ & $36(52.9)$ & $9(64.3)$ & $32(48.5)$ \\
\hline & No & $7(58.3)$ & $32(47.1)$ & $5(35.7)$ & $34(51.5)$ \\
\hline \multicolumn{2}{|c|}{$\mathbf{P}$} & \multicolumn{2}{|c|}{0.471} & \multicolumn{2}{|c|}{0.283} \\
\hline *Fisher's Exact Test & & & & & \\
\hline
\end{tabular}

\section{Discussion}

The study results revealed that a higher percentage of females had systolic and diastolic hypertension than males whereas the mean systolic and diastolic blood pressure was also higher in females. The study findings also showed that the site of headache was positively correlated with both systolic and diastolic blood pressure in males as well as females. Both the severity of headache and the severity of dyspnea were found to be positively correlated with diastolic blood pressure only in males whereas the type of vision problem was negatively correlated with diastolic blood pressure only in females.

Moreover, the study results further displayed that among males the clinical history of headache, vertigo and dyspnea were significantly associated with systolic blood pressure where those who had stage 1 /stage 2 systolic hypertension were more likely to have these symptoms whereas among females only age was significantly associated with diastolic blood pressure where those who had stage 1 /stage 2 diastolic hypertension were more likely to be $\leq 40$ years of age.

Interestingly, a positive correlation between both systolic and diastolic hypertension and the site of headache was revealed by the study results in both genders. As no relevant recent data were available, this finding could not be compared with previous literature.

The study results further showed that, apart from the site of headache, the only symptoms that were positively associated with either systolic or diastolic blood pressure in males were the presence of clinical history of headache, severity of headache and the severity of dyspnea. An earlier study also reported headache to be significantly more prevalent in hypertensive patients than in normotensive subjects $(\mathrm{p}<0.05)$ but literature revealed contrary findings as well. [11]One study searched did not find headache to be associated with hypertension (OR $1.02,95 \%$ CI 0.79 to 1.30 ) while another did not report any significant difference in the prevalence of headache among hypertensive and normotensive subjects $(38.1 \%$ vs. $39.6 \%, p=0.848)[12,13]$. This difference in findings could be attributed to different methods of blood pressure measurement i.e. direct versus indirect auscultatory method in the former study or to different population characteristics in the later study. Also, unlike this study, the results were reported without stratifying by gender in both the studies.

Moreover among males, vertigo and dyspnea were found to be significantly associated with systolic hypertension. Though no relevant data were available to compare the association found between systolic hypertension and vertigo among males in this study, an earlier study also reported dyspnea to be associated with elevated blood pressure though irrespective of gender of the study participants. [14]

Furthermore, the only symptom that had a correlation, though negative, with either systolic or diastolic blood pressure in females was the type of vision problem. An earlier study did not report any statistical difference between the prevalence of visual disturbances among those with or without hypertension though without consideration for the gender of the study participants $(p=0.539)$. [13]The study results further revealed that among 
females, being of $\leq 40$ years of age was significantly associated with diastolic hypertension. Again due to lack of relevant recent data, this finding could not be compared with previous literature.

The study finding of high prevalence of hypertension among females is in line with published literature though contrary findings have also been reported. [15-17]This difference in findings could be attributed to different age criteria for the study participants or to different population characteristics. The study results also showed the prevalence of sleep apnea to be $46.3 \%$ among male and $41.3 \%$ among female study participants respectively. An earlier study reported it to be $22 \%$ of their sample size [18]. This difference in findings could be attributed to different methods of measuring sleep apnea i.e. Self-reported versus polygraphically defined.

\section{Limitation}

The only limitation of the study was the use of convenient sampling method because of financial and time restraints.

\section{Recommendation}

In the light of the study findings it is recommended that the symptoms identified in this study to be associated with either systolic or diastolic blood pressure in any of the genders need to be specially focused by the health care professionals during hypertension screening and management.

\section{Conclusion}

The symptoms significantly associated with systolic or diastolic hypertension included age, clinical history of headache, its site and severity, vertigo, dyspnea and its severity and type of vision problem although a disparity was found among both genders. These findings broaden our understanding of clinical features associated with both systolic and diastolic hypertension in a Pakistani population.

\section{References}

1. He J, Whelton PK. Epidemiology and prevention of hypertension Med Clin North Am.1997; 81(5):1077-1097.

2. Whelton PK. Epidemiology of hypertension. Lancet. 1994; 344(8915):101-106.

3. Ezzati M, Lopez AD, Rodgers A, Vos T, Danaei G, Aryee M et al Comparative risk assessment collaborative group: selected major risk factors and global and regional burden of disease. Lancet 2002; 360(9859):1347-1360

4. Kearney MP, Whelton M, Reynolds K, Muntner P, Whelton KP, Heet J. Global burden of hypertension: analysis of worldwide data. Lancet 2005; 365(9455): 217-223

5. Kearney PM, Whelton M, Reynolds K, Whelton PK, He J. Worldwide prevalence of hypertension: a systematic review. J Hypertens 2004; 22(1): $11-19$

6. Non-communicable Diseases Country Profile 2014. World Health Organization. 2014
7. Roger VL, Go AS, Lloyd-Jones DM, Benjamin EJ, Berry JD, Borden WB et al. Heart disease and stroke statistics--2012 update: a report from the American Heart Association. Circulation. 2012. 125(1):e2-e220; Doi: 10.1161/CIR.0b013e31823ac046

8. Messerli HF, Williams B, Ritz E. Essential hypertension. Lancet 2007; 370(9587): 591-603

9. Mancia G, Fagard R, Narkiewicz K, Redon J, Zanchetti A, Bohm M, et al. 2013 ESH/ ESC Guidelines for the management of arterial hypertension: The Task Force for the management of arterial hypertension of the European Society of Hypertension (ESH) and of the European Society of Cardiology (ESC). European Heart Journal. 2013;34(28): $2159-2219$

10. Chobanian AV, Bakris GL, Black HR, Cushman WC, Green LA, Izzo JL Jr, et al. Seventh report of the Joint National Committee on Prevention, Detection, Evaluation, and Treatment of High Blood Pressure. Hypertension. 2003; 42(6):1206-52

11. Di Tullio M, Alli C, Avanzini F, Bettelli G, Colombo F, Devoto MA, et al. Prevalence of symptoms generally attributed to hypertension or its treatment: study on blood pressure in elderly outpatients (SPAA). Journal of hypertension. Supplement: official journal of the International Society of Hypertension. J Hypertens Suppl. 1988; 6(1):S87-90.

12. Fuchs FD, Gus M, Moreira LB, Moreira WD, Goncalves SC, Nunes G. Headache is not more frequent among patients with moderate to severe hypertension. Journal of human hypertension. 2003 ;17(11):787

13. Sherif SM, Ahmed ME, Homeida MM. Prevalence of hypertension in an urban community in Sudan. Khartoum Medical Journal. 2012;1(2)

14. Karras DJ, Ufberg JW, Harrigan RA, Wald DA, Botros MS, McNamara RM. Lack of relationship between hypertension-associated symptoms and blood pressure in hypertensive ED patients. The American journal of emergency medicine. 2005; 23(2):106-110

15. Kokiwar PR, Gupta SS, Durge PM. Prevalence of hypertension in a rural community of central India. J Assoc Physicians India. 2012; $60(6): 26-29$

16. Esam MS, Husain AS. Prevalence of Prehypertension and Hypertension in rural Bareilly. Natl J Med Res. 2012; 2(3):291-294

17. Choi KM, Park HS, Han JH, Lee JS, Lee J, Ryu OH, et al. Prevalence of prehypertension and hypertension in a Korean population: Korean National Health and Nutrition Survey 2001. J Hypertens. 2006; 24(8):1515-1521; Doi: 10.1097/01.hjh.0000239286.02389.0f

18. Lavie P, Ben-Yosef R, Ami-hai ER. Prevalence of sleep apnea syndrome among patients with essential hypertension. American heart journal. 1984; 108(2):373-376; Doi: 10.1016/0002-8703(84)90628-8 